\title{
Frequency hopping in IEEE 802.15.4 to mitigate IEEE 802.11 interference and fading
}

\author{
JAVED Umer ${ }^{1}$, HE Di ${ }^{1, *}$, LIU Peilin ${ }^{1}$, and YANG Yueming ${ }^{2}$ \\ 1. Shanghai Key Laboratory of Navigation and Location-based Services, Shanghai Jiao Tong University, Shanghai 200240, China; \\ 2. Shanghai Panchip Microelectronics Co., Ltd, Shanghai 201210, China
}

\begin{abstract}
In this paper, we investigate the issues of initialization and deployment of wireless sensor networks (WSNs) under IEEE $802.11 \mathrm{~b} / \mathrm{g}$ interference and fading channels using frequency hopping $(\mathrm{FH})$. We propose an $\mathrm{FH}$ algorithm for WSNs, which is implemented and tested with a pair of nodes employing IPv6 over low power wireless personal area networks (6LoWPAN) standard. The merits and demerits of the proposed FH scheme in WSNs are studied under strong IEEE $802.11 \mathrm{~b} / \mathrm{g}$ interference and frequency selective fading channels. We compare the performance results of the proposed $\mathrm{FH}$ scheme with those obtained by single-channel radio in WSNs, and show that FH maintains very reliable data rates in the presence of adverse conditions where the single-channel radio fails. We determine a minimum center frequency offset of channels between IEEE 802.15.4 and IEEE 802.11b/g-based networks, which guarantees the error free network operation of IEEE 802.15.4 using a single channel. We design a second $\mathrm{FH}$ procedure comprising only four free channels $(15,20,25$, and 26$)$ of IEEE 802.15.4 standard, and show that in the presence of nearby IEEE $802.11 \mathrm{~b} / \mathrm{g}$ interference, the IEEE 802.15 .4 data rate using this method is always $98 \%$ and more.
\end{abstract}

Keywords: IEEE 802.15.4, wireless sensor networks (WSNs), frequency hopping $(\mathrm{FH})$, interference avoidance, coexistence.

DOI: $10.21629 /$ JSEE.2018.03.01

\section{Introduction}

The $2.4 \mathrm{GHz}$ industrial, scientific and medical (ISM) band is allocated to numerous wireless standards in addition to IEEE 802.15.4 [1] for wireless sensor networks (WSNs). The IEEE $802.11 \mathrm{~b} / \mathrm{g}$ standard [2] for wireless data access occupies the ISM band. Bluetooth devices and wireless

\footnotetext{
Manuscript received December 12, 2016.

*Corresponding author.

This work was supported by the Important National Science and Technology Specific Project of China (2018ZX03001020-005; 2016ZX03001022-006), the National Natural Science Foundation of China (61771308), the Shanghai Science and Technology Committee (16DZ1100402), and the Special Project of Military and Civilian Integration of Shanghai Economic and Information Committee with the name "Ultra High Sensitivity UAV Communication System Based on Radar Related Technology".
}

telephones are also deployed in this ISM frequency band, there might also be unknown jammers operating on the same or nearby frequency [3-8]. These wireless systems are located close to each other in actual network deployments, with heterogeneous signal powers corresponding to different wireless standards. Therefore, WSNs must be able to coexist with these wireless systems and operate reliably [9-13]. Interference from other wireless systems and intentional jamming from dedicated jammers is one of the major sources of unreliability in WSN. The second major cause of rapid packet drop in WSN is multipath fading [14-21]. A deep fade in the channel can cause complete data communication failure for some time. In this work we make use of frequency hopping $(\mathrm{FH})$ to solve the above stated problems of interference and fading in WSN.

Now we go through a short review of previous work done on multi-carrier communication in WSNs. The problem of coexistence of IEEE 802.15.4 with IEEE $802.11 \mathrm{~b}$ interference was studied in [22] and two adaptive channel allocation methods were given. Dynamic performance evaluation of IEEE 802.15.4 network with many radio frequency (RF) interferences was reported in [23]. Performance analysis of IEEE 802.15.4 physical layer (PHY) with wireless channel impulsive interference modeling was provided in [24]. In [25] effect of interference in adjacent and alternate channels was quantified in terms of packet drop ratio by varying different parameters. The work in [26] presented a study of the time slotted channel hopping IEEE 802.15.4, part of the 6TiSCH stacked and described how its time division multiple access (TDMA) method increased the reliability with performance guarantees. Performance evaluation and improvement of TCP/IPv6 in IEEE 802.15.4 with IEEE 802.11 interference were reported in [27]. The work in [28] using measurements in a real-life testbed, gave a comparison of different channel selection methods based on a multichannel protocol classification. A new cooperation method called cooperative channel segmentation (CCS) was proposed in 
[29], between IEEE 802.11 wireless local area network (WLAN) and bluetooth based wireless personal area network (WPAN), which operates in the $2.4 \mathrm{GHz}$ ISM band. A game theory-based power allocation mechanism was proposed in [30] for IEEE 802.11 networks in ISM bands, which might incorporate some aspects of cognitive radio functionality. A cross-layer design that used PHY characteristics of 802.15.4 signals to deal with cross-technology interference was investigated in [31]. Nowadays, standards [32 - 34] governing WSNs also recommend using $\mathrm{FH}$ in media access control (MAC) layer as countermeasure of strong interference.

The main aim of this work is to develop FH algorithms to deal with two critical problems in WSNs: (i) We develop, implement and evaluate $\mathrm{FH}$ algorithms to mitigate the interference in an IEEE 802.15.4 network from IEEE $802.11 \mathrm{~b} / \mathrm{g}$ device and analyze the performance of the proposed algorithms; (ii) We use FH to avoid and minimize the multipath fading (by converting it into narrowband flat fading) in $2.4 \mathrm{GHz}$ frequency band, and analyze the perfor- mance of the proposed algorithms in different fading channels.

The rest of this paper is organized as follows: Section 2 gives the description of our FH algorithm, Section 3 describes the usefulness of FH against IEEE $802.11 \mathrm{~b} / \mathrm{g}$ interference, Section 4 shows the effects of center frequency offsets between the channels of IEEE 802.15.4 and IEEE $802.11 \mathrm{~b} / \mathrm{g}$, based on these results a new channel selection for FH pattern is defined, and Section 5 explains the effectiveness of FH in cancelling effects of frequency selective fading channels.

\section{FH algorithm}

The channel allocations of IEEE 802.11b (non-overlapping sets) and IEEE 802.15.4 in 2.4 GHz ISM band are shown in Fig. 1. It can be observed that four channels $(15,20,25$, and 26) of IEEE 802.15.4 lie on the empty bands of IEEE 802.11b channel allocation. If IEEE 802.15.4 devices are operating in these free bands, then there will be no interference from IEEE $802.11 \mathrm{~b}$ as compared to the remaining channels.

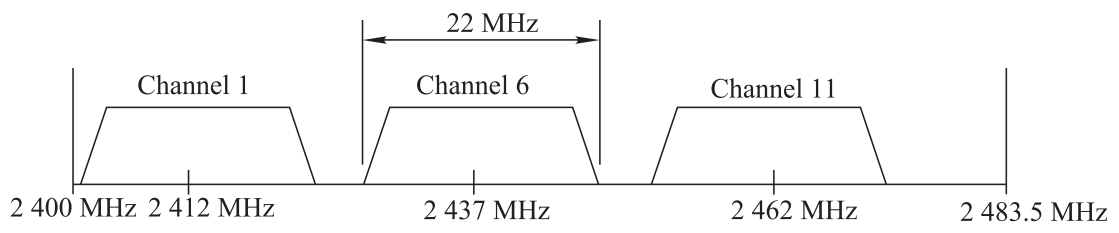

(a) IEEE 802.11b North American channel selection (nonoverlapping)

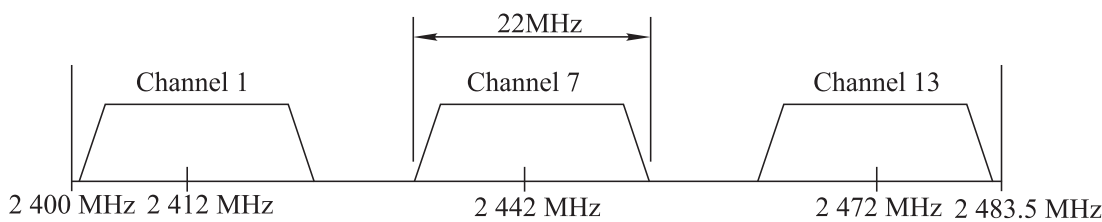

(b) IEEE 802.11b European channel selection (nonoverlapping)

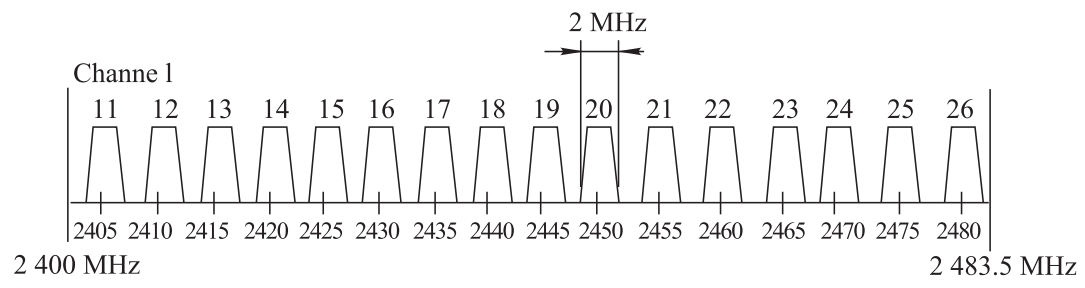

(c) IEEE 802.15.4 channel selection (2 $400 \mathrm{MHz}$ PHY)

Fig. 1 IEEE 802.15.4 and IEEE 802.11b channel selection [1]

In this section, the proposed FH algorithm for WSNs is described in detail, its theoretical background is given, and its efficiency and computational complexity are discussed. The same FH scheme is used later in the developed test beds to demonstrate its efficacy in various scenarios.

\subsection{Algorithm}

One of the challenges of any FH system is to first time synchronize the transmitter and receiver, before data exchange on designated channels could be started. Our proposed FH algorithm is shown in Fig. 2. When a child node has data to send to a root node (cluster head $(\mathrm{CH})$ ) and vice versa, it first must be time synchronized to a root node and then transmit data based on some channel hopping sequence. The synchronization method used in this work is timing-sync protocol for sensor networks (TPSN) 
[35]. TPSN is a sender-receiver synchronization protocol, so it is best suited for WSNs where the sensor node (here the child node) having the required information initiates the communication with the $\mathrm{CH}$ (here the root node). For details on the working principle of TPSN, see Sections 4 and 5 in [35]. Details on a real-world deployment of TPSN on standalone sensor nodes without having any additional components are also presented in [35]. After both the child and root nodes have been synchronized, the channel hopping phase starts. In the next step of FH, as shown in Fig. 2, the root node sends a data packet to a child node containing a random $\mathrm{FH}$ sequence of 16 channels or less than 16, a hop interval time (time to stay at one channel) of $1 \mathrm{~s}$ or less and the frequency hopping start time. To obtain FH pattern, a sequence of uniformly distributed pseudorandom numbers from an interval $[1,16]$ is generated using a uniform random number generator. It is important to note that the synchronization phase and initialization steps are executed on a predetermined secure channel or a set of channels, so the availability and quality of radio communication on this radio channel is of critical importance. If the synchronization phase and initialization steps are not completed due to some problems, then they are repeated until their successful completion. In the third step of FH, both nodes hop on the same channel at the same time and data is transferred from the child node to the root node and vice versa. A loose or tight time synchronization can be maintained according to the system requirements, as our method supports both requirements. Here in Fig. 2, loose time synchronization is shown, as synchronization is not repeated during FH data transfer. The maximum time for $\mathrm{FH}$ is $16 \mathrm{~s}$ for 16 channels and is always less than $16 \mathrm{~s}$ when combinations of fewer channels (less than 16) and small hop interval time (less than $1 \mathrm{~s}$ ) are used. It is quantified as

FH time $=$ number of channels $\times$ hop interval time,

number of channels $\leqslant 16$, hop interval time $\leqslant 1 \mathrm{~s}$.

We observe that there is no packet loss with loose time synchronization due to negligible clock drift between the two nodes for $16 \mathrm{~s}$. Repeated measurements are performed and analyzed to ensure this hypothesis of loose synchronization is correct. After the hopping pattern (e.g., $[15,11,20,19,25,23,14$ and 26]) has been traversed for one time, the two nodes must go to synchronization phase and then to FH phase in order to maintain sufficient time synchronization. A new channel hopping pattern is generated using a uniform random number generator each time, after the last channel sequence has been used. This synchronization-FH-synchronization loop in Fig. 2 continues until the child node has data to transmit to the root node and vice versa. The synchronization-FHsynchronization loop ends when there is no data to exchange and the nodes go to an idle mode. If tight time synchronization is required, then this can be achieved by sending each packet on a different channel, and repeating synchronization after reception of every packet. Thus, the FH technique is completed by performing packet transmission and time synchronization alternately in a sequence.

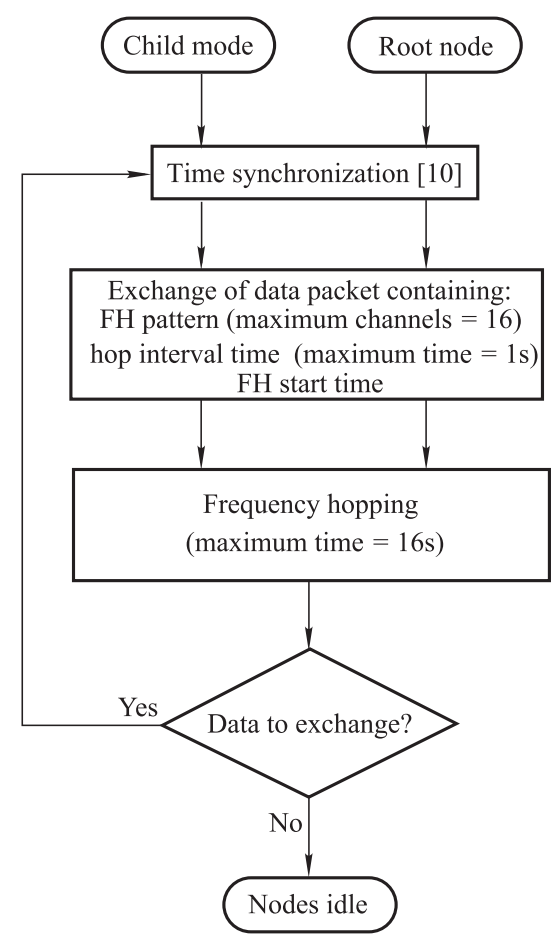

Fig. 2 An FH algorithm for IEEE 802.15.4

The proposed FH algorithm is very efficient and its computational complexity is very low. The only extra cost required compared to single channel operation is in terms of time and energy required for channel switching and synchronization. However, the modern sensor nodes are equipped with very fast and efficient processors, which execute these tasks in nearly no time and with minimal energy usage.

\subsection{Signaling and channels}

The FH procedure proposed in this work needs signaling channel for time synchronization phase and information (hopping pattern, hop interval time and FH start time) exchange related to $\mathrm{FH}$, before $\mathrm{FH}$ successfully starts. This pre-FH phase is communicated through a default channel known to all network nodes. The selection of a default channel is very important. If it is continuously interfered or in deep fade (which is rare), then this FH procedure cannot be established. The criteria for default channel selection vary with applications, for which WSN is being 
used. It also depends on the operating conditions of the network. We assume that nodes periodically update their default channel by employing a particular channel selection algorithm. If a given WSN has cognitive radio (CR) capability, then many standard spectrum sensing techniques can be used to determine the best channel for initialization phase.

\section{FH in IEEE 802.15.4 as a counter measure against IEEE $802.11 \mathrm{~b} / \mathrm{g}$ interference}

The proposed FH scheme is implemented on Sensinode 6LoWPAN Devkit [36] to evaluate its performance under different test conditions. The Devkit is equipped with the Devboard, which is used for programming and debugging sensor nodes. The software product included is Sensinode's NanoStack which implements the 6LoWPAN commercial protocol stack. The FH scheme is written on application, network and data link layers of NanoStack as shown in Fig. 3. In the rest of this section testbed description is given thoroughly and performance analysis of the $\mathrm{FH}$ algorithm is explained in full detail.

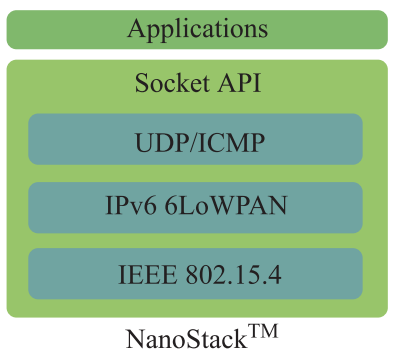

Fig. 3 Sensinode's NanoStack

\subsection{Testbed description}

The testbed developed for the performance evaluation of the proposed $\mathrm{FH}$ algorithms with interference is shown in Fig. 4. When transmitting the data without the need for monitoring the transmitter, the computer and development board are not required. The nodes can be programmed with different applications by using the development board. The nodes are powered by batteries or connecting to the development board and computer. When receiving the data, the computer and development board are required for recording the statistics of the received data packets. The parameters of the testbed applicable to all measurements and emulations are shown in Table 1.

The interferer is built by connecting an external IEEE 802.11 b card to a laptop computer. It is possible to change the amount of transmitted power and the frequency of transmitted signals. Multi-generator (MGEN) [37] is used as a data source for the IEEE 802.11b interferer. MGEN can generate different types of real-time user traffic and supports the unicast and multicast modes of IEEE 802.11b.

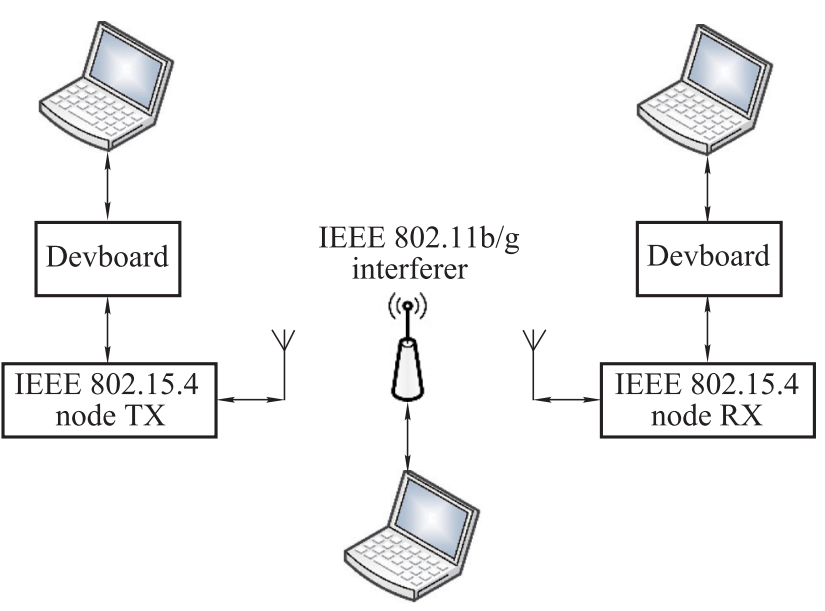

Fig. 4 Interference analysis testbed

Table 1 Testbed parameters

\begin{tabular}{cc}
\hline Parameter & Value \\
\hline IEEE 802.15.4 packet size/bytes & 128 \\
IEEE 802.15.4 output power/dBm & 0 \\
IEEE 802.15.4 clear channel assessment (CCA) mode & 2 \\
IEEE 802.15.4 packet inter-transmission time/ms & 10 \\
IEEE 802.11g packet rate/(packets/s) & 1000 \\
IEEE 802.11g packet size/bytes & 1472 \\
IEEE 802.11g output power/dBm & 16.5 \\
\hline
\end{tabular}

\subsection{Results and analysis}

In this section we describe the experimental results obtained from the developed testbed, these results quantify the performance of IEEE 802.15.4 network in the presence of strong IEEE $802.11 \mathrm{~b}$ interference in different office environments. To fully show the impact of IEEE $802.11 \mathrm{~b}$ interference, the transmit power of interfering signals is set at maximum. The interfering signals occupy the nonoverlapping channels used in North America. There are also other IEEE $802.11 \mathrm{~b}$ networks present outside the office environment where the experiments have been performed, which occupy the non-overlapping channels used in Europe. Thus, these unintentional interference sources having low to medium signal powers may possibly affect the results of the testbed. The channel selections for North America and Europe are explained in Section 1. The channel activity of IEEE $802.11 \mathrm{~b}$ transmitter is $78 \%$, where the length of the transmitted data is 1500 bytes. The signals of both networks (IEEE 802.15.4 and IEEE 802.11b/g) experience multipath fading as they are deployed in an indoor office environment. The IEEE $802.11 \mathrm{~b}$ transmitter is emitting signals on a specific channel and this channel 
is changed according to the requirements of experiments. The performance of IEEE 802.15.4 network is measured by the packet delivery ratio (PDR). The PDR is calculated by

$$
\operatorname{PDR}(\%)=\frac{(\text { No. of the received packets })}{(\text { No. of the transmitted packets })} \times 100 \text {. }
$$

It is important to mention that the considered performance evaluation metric, that is, PDR is the most widely used for measurement and analysis at data link, network and transport layers. Thus, PDR can be easily translated into PHY metrics such as symbol and bit error rate. Also, packet level information is always available in all the WSNs based on IEEE 802.15.4. Therefore, this work is based on PDR metric as its scope is broad and also it is the most used one for measuring the amount of data delivered successfully.

When an IEEE 802.15.4 network operates on a single channel, the measurement results are shown in Table 2. As the IEEE 802.15.4 network operates on a single channel, the resulting PDR is too small and does not fulfill the re- quirement of reliable data delivery. In the first case, the distance of the IEEE 802.11b interferer from IEEE 802.15.4 network is $1 \mathrm{~m}$ and two IEEE 802.15.4 nodes are located at the distance of $1 \mathrm{~m}$ from each other. Although the sensor nodes are close to each other, the achieved PDR is 50\% due to very strong interference, this PDR is considered low for reliable operation of WSNs. Therefore, the PDR of IEEE 802.15.4 network will not increase with small distance between the nodes, when a source of strong interference is also located near to them. In the second case, the distance of the IEEE $802.11 \mathrm{~b}$ interferer from IEEE 802.15.4 network is $1 \mathrm{~m}$ and two IEEE 802.15.4 nodes are located at the distance of $10 \mathrm{~m}$ from each other. The achieved PDR is $10 \%$ due to the extremely strong interference, as the distance between the transmitter and receiver has increased much now, so the same level of interference causes more packet loss in the second case. Thus, it is concluded that IEEE 802.15.4 networks should not be deployed using only a single channel, especially in unknown or hostile environments, otherwise the wireless network operation will fail completely most of the times.

Table 2 Performance analysis of single channel IEEE 802.15.4

\begin{tabular}{cc}
\hline Channel activity of IEEE $802.11 \mathrm{~b} / \%$ & 78 \\
Distance of IEEE 802.15 .4 receiver from IEEE $802.11 \mathrm{~b}$ interferer/m & 1 \\
Channel of IEEE $802.11 \mathrm{~b}$ & 1 \\
Channel of IEEE 802.15 .4 & 12 \\
\hline Carrier frequency offset between IEEE 802.11b and IEEE 802.15 .4 signals $/ \mathrm{MHz}$ & 2 \\
\hline Distance between two IEEE 802.15 .4 devices $=1 \mathrm{~m}$ & Distance between two IEEE 802.15 .4 devices $=10 \mathrm{~m}$ \\
\hline PDR (\%) of IEEE $802.15 .4=50 \pm 1$ & PDR (\%) of IEEE $802.15 .4=10 \pm 1$ \\
\hline
\end{tabular}

When an IEEE 802.15.4 network uses the FH spread spectrum, the measurement results are shown in Table 3 and Table 4. We can see a huge increase in PDR with FH in comparison with Table 2 for a network using a single channel. When two IEEE 802.15.4 nodes are located $1 \mathrm{~m}$ apart, the PDR attained is $88 \%, 94 \%$ and $96 \%$ for IEEE 802.11b interfering signals transmitted on channels 1,6 and 11, respectively. It is observed that for different channels of IEEE $802.11 \mathrm{~b}$ interferer there is a little difference in PDR. If we compare it with Table 2 for a network using a single channel, the noticeable increase in PDR is $38 \%$, $44 \%$ and $46 \%$.

Table 3 Performance analysis of IEEE 802.15.4 using FH while the distance between two devices is set to $1 \mathrm{~m}$

\begin{tabular}{|c|c|c|}
\hline \multicolumn{2}{|c|}{ Channel activity of IEEE $802.11 \mathrm{~b} / \%$} & 78 \\
\hline \multicolumn{2}{|c|}{ Distance of IEEE 802.15 .4 receiver from IEEE $802.11 \mathrm{~b}$ interferer $/ \mathrm{m}$} & 1 \\
\hline \multicolumn{2}{|c|}{ Distance between two IEEE 802.15 .4 devices $/ \mathrm{m}$} & 1 \\
\hline Channel of IEEE $802.11 \mathrm{~b}=1$ & Channel of IEEE $802.11 \mathrm{~b}=6$ & Channel of IEEE $802.11 \mathrm{~b}=11$ \\
\hline PDR $(\%)$ of IEEE $802.15 .4=88 \pm 2.00$ & PDR $(\%)$ of IEEE $802.15 .4=94 \pm 0.55$ & PDR $(\%)$ of IEEE $802.15 .4=96 \pm 0.18$ \\
\hline
\end{tabular}

Table 4 Performance analysis of IEEE 802.15.4 using FH while the distance between two devices is set to $10 \mathrm{~m}$

\begin{tabular}{ccc} 
Channel activity of IEEE $802.11 \mathrm{~b} / \%$ & 78 \\
Distance of IEEE 802.15 .4 receiver from IEEE $802.11 \mathrm{~b}$ interferer $/ \mathrm{m}$ & 1 \\
Distance between two IEEE 802.15 .4 devices $/ \mathrm{m}$ & Channel of IEEE $802.11 \mathrm{~b}=11$ \\
\hline Channel of IEEE $802.11 \mathrm{~b}=1$ & Channel of IEEE $802.11 \mathrm{~b}=6$ & PDR $(\%)$ of IEEE $802.15 .4=80 \pm 0.55$ \\
\hline PDR $(\%)$ of IEEE $802.15 .4=80 \pm 0.91$ & PDR $(\%)$ of IEEE $802.15 .4=87 \pm 1.00$ & 0 \\
\hline
\end{tabular}


Now we explain the factors which affect the FH system, when its PDR is $88 \%$ instead of being more than $90 \%$. Some channels of FH system experience fading, so this multipath fading decreases PDR over these specific frequencies. All IEEE $802.11 \mathrm{~b}$ access points in the surroundings of the test environment are operating on channel frequencies of Europe. The PDR of $88 \%$ represents real-time scenarios when the transmitted signals are affected simultaneously by the interference and flat fading. When the interference and fading occur together then they affect the signal transmission in two ways. The fading is only present on the interfered channels and does not affect the channels free from interference: in this case, the interfered channels suffer from packet loss already and the detrimental effect of fading is not much. In the second case, the interference is affecting some set of channels, while the signal fading is occurring on the other channels not in this set. This second case will cause a little decrease in the effectiveness of FH, when FH is not used together with adaptive or cognitive radio system. The PDR of $88 \%$ results from the channel conditions described in the second case.

As shown in Table 4, when two IEEE 802.15.4 nodes are located $10 \mathrm{~m}$ apart, the PDR attained is $80 \%, 87 \%$ and $80 \%$ for IEEE $802.11 \mathrm{~b}$ interfering signals transmitted on channels 1, 6 and 11, respectively. If we compare it with Table 2 for a network using a single channel, the noticeable increase in PDR is 70\%, 77\% and 70\%. These results show that the effectiveness of FH increases even more when the sensor nodes are located far apart and the transmitter is close to the interferer. Our results clearly demonstrate that IEEE 802.15.4 network can attain performance and reliability of more than $85 \%$ in all scenarios, when the proposed fast FH algorithm is used for network operation.

The theoretical basis of frequency-hopping spread spectrum (FHSS) is well established in the previous literature. Therefore, the theoretical performance bounds (minimum or maximum) derived for a general FHSS communication system in various conditions are also applicable to our proposed algorithm. In this work, our goal and emphasis are to give the performance evaluation of proposed FH methods in practical scenarios and prove their effectiveness in real-time.

\section{Carrier frequency offset between signals of IEEE 802.15.4 and IEEE 802.11b}

Here we provide novel results and corresponding analysis regarding the effect of carrier frequency offset between signals of IEEE 802.15.4 and IEEE 802.11b networks. Based on this analysis, we also develop robust versions of $\mathrm{FH}$ methods described in the previous sections.

\subsection{Testbed results and analysis}

Fig. 5 and Fig. 6 show the relationship between the PDR of IEEE 802.15.4 and the carrier frequency offset between the IEEE 802.15.4 signal and interference. It is seen from Fig. 5 that if the carrier frequency offset between IEEE 802.15.4 and IEEE $802.11 \mathrm{~b}$ is greater than $7 \mathrm{MHz}$, the IEEE 802.15.4 PDR exceeds 83\% giving a reliable sensor network. In case of Fig. 6, PDR is between $55 \%$ for $8 \mathrm{MHz}$ offset and greater than $90 \%$ after that. These novel theoretical and experimental results are reported for the first time here in this work, which clearly show that the carrier frequency difference between IEEE 802.15.4 and IEEE $802.11 \mathrm{~b} / \mathrm{g}$ based networks should be larger than $7 \mathrm{MHz}$ for interference free operation of IEEE 802.15.4 based WPANs. From the results it is confirmed that if there is a closely located IEEE $802.11 \mathrm{~b}$ transmitter ( $1 \mathrm{~m}$ in our case), and the carrier frequency difference is more than $7 \mathrm{MHz}$, then the PDR of IEEE 802.15.4 network will be greater than $90 \%$. FH has the inherent gain, that the transmitted signals in FH occupy those channels for very short time which do not satisfy the carrier frequency offset condition of more than $7 \mathrm{MHz}$.

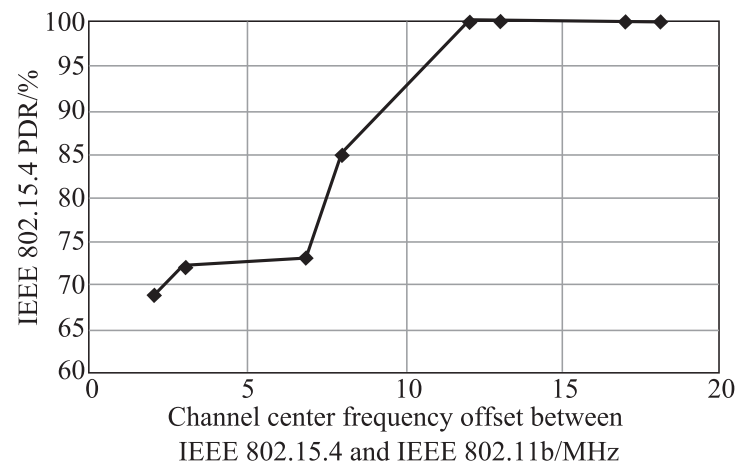

Fig. 5 Carrier frequency offset (distance between two IEEE 802.15.4 devices is $1 \mathrm{~m}$ )

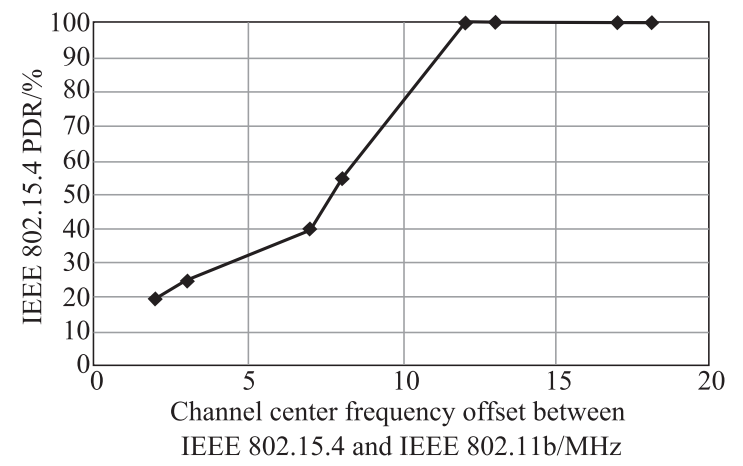

Fig. 6 Carrier frequency offset (distance between two IEEE 802.15.4 devices is $10 \mathrm{~m}$ )

\subsection{FH in IEEE 802.15.4 using free channels $(15,20,25,26)$}

We propose an $\mathrm{FH}$ procedure based on channel selection 
information described in Section 1 and results from Fig. 5 and Fig. 6. This procedure comprises only four free channels $(15,20,25$, and 26). The results are provided in Table 5 and Table 6 . For the first system in Table 5, if the two IEEE 802.15.4 devices are $1 \mathrm{~m}$ apart, the attained PDR is $98 \%, 98 \%$ and $99 \%$, when IEEE $802.11 \mathrm{~b}$ transmitter occupies channels 1,6 and 11 , respectively. If we compare
Table 3 and Table 5, we see the IEEE 802.15.4 PDR improvement of $10 \%, 4 \%$ and $3 \%$. For the second system in Table 6, if the two IEEE 802.15.4 devices are $10 \mathrm{~m}$ apart, the attained PDR is $98 \%, 99 \%$ and $99 \%$, when IEEE $802.11 \mathrm{~b}$ transmitter occupies channels 1,6 and 11 , respectively. If we compare Table 4 and Table 6 , we see the IEEE 802.15.4 PDR improvement of $18 \%, 12 \%$ and $19 \%$.

Table 5 Performance analysis of IEEE 802.15.4 using FH (channels $=15,20,25,26$ ) while the distance between two devices is set to $1 \mathrm{~m}$

\begin{tabular}{ccc}
\hline Channel activity of IEEE $802.11 \mathrm{~b} / \%$ & 78 \\
Distance of IEEE 802.15.4 receiver from IEEE $802.11 \mathrm{~b}$ interferer $/ \mathrm{m}$ & 1 \\
Distance between two IEEE 802.15 .4 devices $/ \mathrm{m}$ & Channel of IEEE $802.11 \mathrm{~b}=11$ \\
\hline Channel of IEEE $802.11 \mathrm{~b}=1$ & Channel of IEEE $802.11 \mathrm{~b}=6$ & PDR $(\%)$ of IEEE $802.15 .4=99 \pm 1$ \\
\hline PDR $(\%)$ of IEEE $802.15 .4=98 \pm 0.56$ & PDR $(\%)$ of IEEE $802.15 .4=98 \pm 1.46$ & \\
\hline
\end{tabular}

Table 6 Performance analysis of IEEE 802.15.4 using FH (channels $=15,20,25,26$ ) while the distance between two devices is set to $10 \mathrm{~m}$

\begin{tabular}{ccc} 
Channel activity of IEEE $802.11 \mathrm{~b} / \%$ & 78 \\
Distance of IEEE 802.15 .4 receiver from IEEE $802.11 \mathrm{~b}$ interferer $/ \mathrm{m}$ & 1 \\
Distance between two IEEE 802.15 .4 devices $/ \mathrm{m}$ & Channel of IEEE $802.11 \mathrm{~b}=11$ \\
\hline Channel of IEEE $802.11 \mathrm{~b}=1$ & Channel of IEEE $802.11 \mathrm{~b}=6$ & PDR $(\%)$ of IEEE $802.15 .4=99 \pm 1$ \\
\hline PDR $(\%)$ of IEEE $802.15 .4=98$ & PDR $(\%$ IEEE $802.15 .4=99 \pm 0.97$ & \\
\hline
\end{tabular}

The above results verify the effectiveness of the reduced channel set containing four free channels. This method works maximally when the interferer is IEEE $802.11 \mathrm{~b}$ and uses the non-overlapping channel selection explained in Section 1 . The proposed method may not work effectively when the interferer is not IEEE $802.11 \mathrm{~b}$ complaint, and if these four free channels are experiencing deep fade.

The interference level in these test beds can be varied from low to high as required in a particular case. The results and analyses presented here are obtained with the most commonly encountered interference levels, as the transmit powers of IEEE $802.11 \mathrm{~b} / \mathrm{g}$ based WLANs vary in a fixed range. The PDR will be low for higher interference levels, and high for low interference levels, if all the other parameters of the considered FH methods are kept the same during the performance evaluation.

\section{FH in frequency selective fading channels}

In this section we discuss the operation of $\mathrm{FH}$ in fading channels and compare results to IEEE 802.15.4 based on a single channel. The main motivation of using FH here is its proven effectiveness against dispersive fading models (frequency selective). FH operation has a signal bandwidth of $2 \mathrm{MHz}$ in IEEE 802.15.4, which converts the frequency selective fading channel into narrow band flat fading channel.

\subsection{Testbed description}

The testbed of Fig. 7 is used for evaluating the performance of FH in the multipath Rayleigh \& Rician fading channel models. This digital signal transmission system has some components which are the same as in the first system shown in Fig. 4. A radio channel emulator acts as a wireless channel between the transmitter and receiver. Since the channel emulator has two one-way channels, circulators transform these channels into a two-way channel. We use the wireless channel models of [38] and [39]. These models cover the $2 \mathrm{GHz}$ and $5 \mathrm{GHz}$ frequency bands of the radio spectrum.

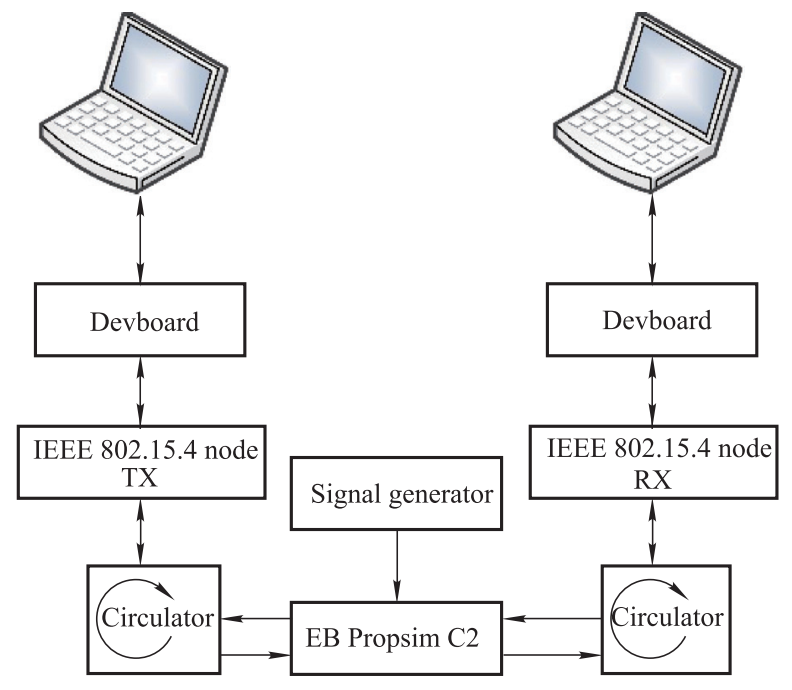

Fig. 7 Multipath fading emulator testbed

\subsection{Channel models}

We give a short description of channel models and their parameters in Table 7. For details refer to [38] and [39]. 
Channel models are constructed inside the channel emulator by inserting the values of the following parameters in the form of tables: tap index $(1-24)$, excess delay $(0-$ $400000 \mathrm{~ns}$ ), power (relative path attenuation) $(\mathrm{dB})$, delay profile (constant), fading profile (Rayleigh and Rice), Doppler spectrum (classical), Doppler shift $(6.7083 \mathrm{~Hz})$, mobile speed $(3 \mathrm{~km} / \mathrm{h})$. The reader can refer to [40] for details on PHY principles of FH, including fading and diversity.

Table 7 Parameters of channel models [41]

\begin{tabular}{cccc}
\hline Model & $\begin{array}{c}\text { R.M.S. delay } \\
\text { spread/ns }\end{array}$ & Fading & Environment \\
\hline A & 50 & Rayleigh & $\begin{array}{c}\text { Office no line of } \\
\text { sight (NLOS) } \\
\text { Open space/office } \\
\text { B }\end{array}$ \\
C & 100 & Rayleigh & $\begin{array}{c}\text { NLOS } \\
\text { Large open space } \\
\text { NLOS }\end{array}$ \\
D & 140 & Rayleigh & $\begin{array}{c}\text { Large open space } \\
\text { line of sight (LOS) }\end{array}$ \\
\hline
\end{tabular}

\subsection{Results and analysis}

Here we give the results and corresponding analysis of $\mathrm{FH}$ in fading channels of Table 7. All the channels of IEEE 802.15.4 occupy $83.5 \mathrm{MHz}$ in the $2.4 \mathrm{GHz}$ band. The bandwidth of the radio channel emulator is $30 \mathrm{MHz}$. Thus, to overcome this constraint we use five channels $(11,12,13$, $14,15)$ of $30 \mathrm{MHz}$ bandwidth instead of all 16 channels. In Figs. 8 -11, the frequency response of the channels is plotted when the signals occupy a total bandwidth of $100 \mathrm{MHz}$. Thus, the frequency response of five channels occupying $30 \mathrm{MHz}$ bandwidth, and also the frequency response of 16 channels occupying $83.5 \mathrm{MHz}$ bandwidth are shown. In these figures, the 0 frequency is equal to the carrier frequency of the five channels, which is the carrier frequency of channel $13(2415 \mathrm{MHz})$. In these figures, the frequency range of $-1.5 \times 10^{7} \mathrm{~Hz}$ to $1.5 \times 10^{7} \mathrm{~Hz}$ is equal to the frequency range of the five channels in $2400-2430 \mathrm{MHz}$. Also, for all 16 channels, the 0 frequency represents the carrier frequency of $2441.75 \mathrm{MHz}$ and the range of frequencies is $-4.175 \times 10^{7} \mathrm{~Hz}$ to $4.175 \times 10^{7} \mathrm{~Hz}$ on the frequency response.

\subsubsection{Channel Model A}

The PDR of $85 \%$ in a single channel, as presented in Table 8, improves to 95\% in FH, as presented in Table 9. The increase in PDR with FH is $10 \%$. This channel model is good in 2 400-2 $430 \mathrm{MHz}$ frequency band of $30 \mathrm{MHz}$ bandwidth. There are two fades in the frequency response: one is deep and the other is small. Only these two fades affect the signal transmission using a single channel, so the resulting PDR is $85 \%$. The PDR with $\mathrm{FH}$ is $95 \%$ which is excellent, because FH enables the transmitted signals to avoid frequencies experiencing fading. When signals are transmitted using 16 channels with $83.5 \mathrm{MHz}$ bandwidth, then in this frequency range two more deep fades are presented. Thus, the PDR would be near $70 \%$ with a single channel and in 90\%-95\% range with FH.

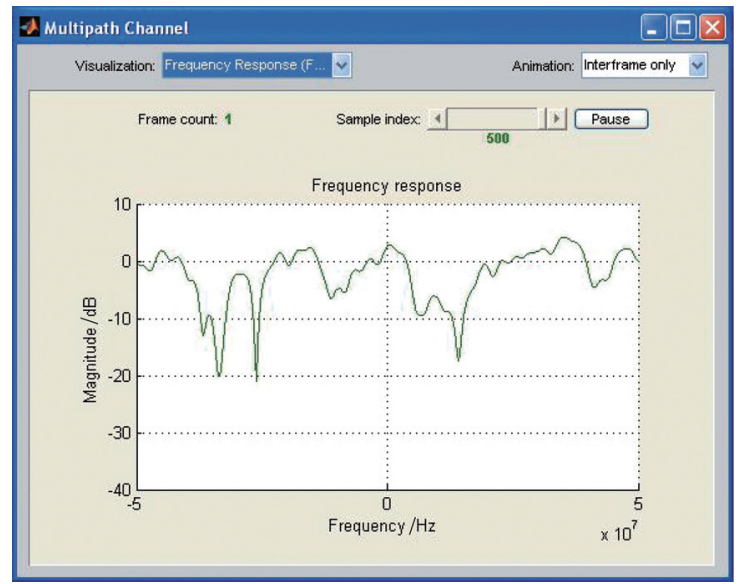

Fig. 8 Frequency response of Model A

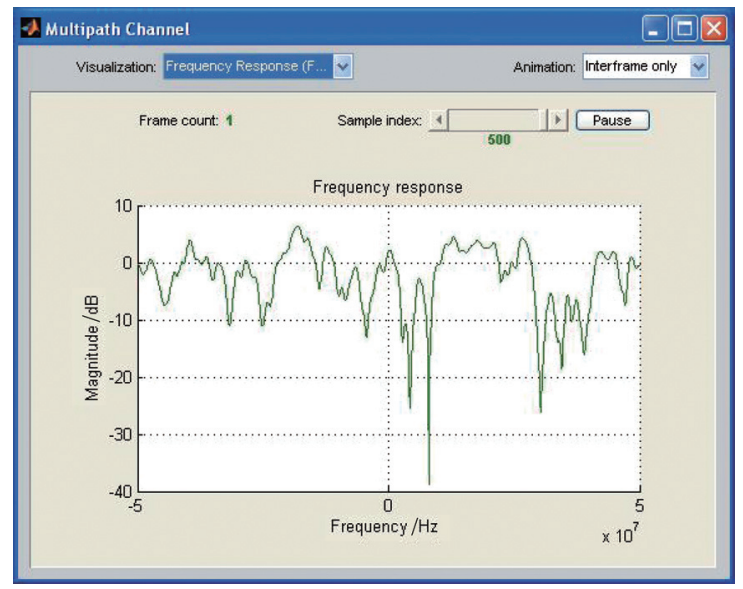

Fig. 9 Frequency response of Model B

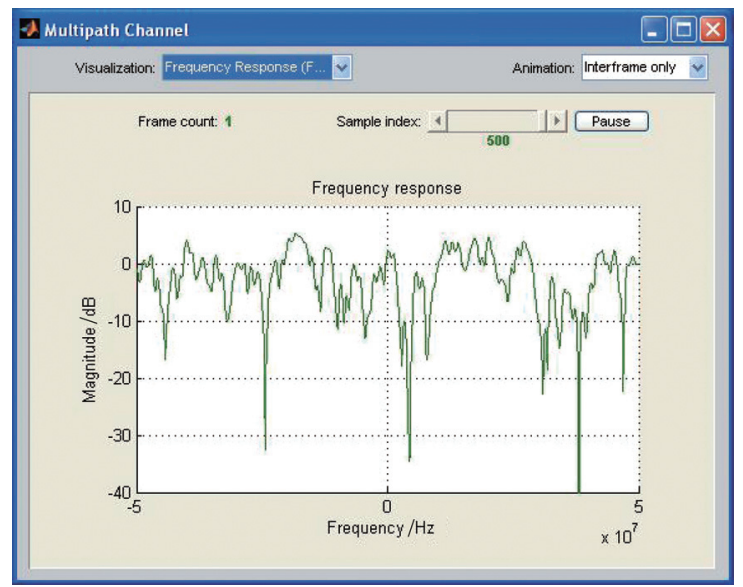

Fig. 10 Frequency response of Model C 


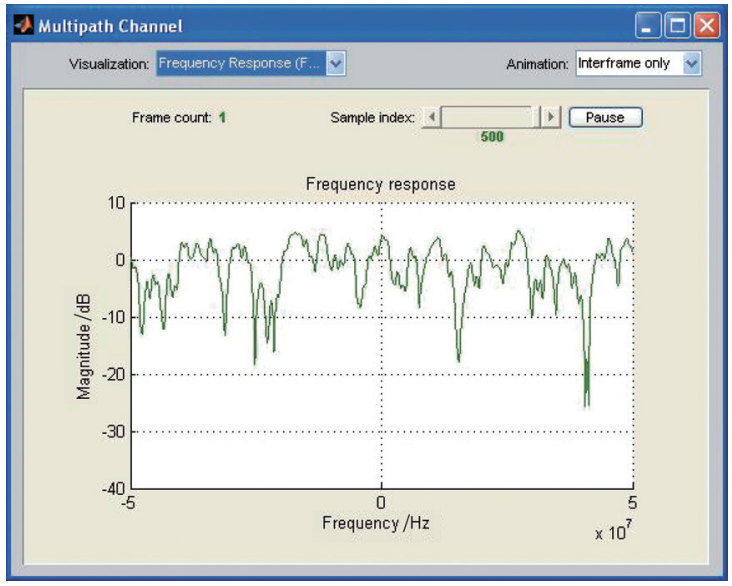

Fig. 11 Frequency response of Model D

Table 8 Testbed results with single channel

\begin{tabular}{cc}
\hline Channel model & IEEE 802.15.4 PDR/\% \\
\hline A & $85 \pm 1$ \\
B & $77 \pm 2$ \\
C & $70 \pm 2$ \\
D & $80 \pm 2$ \\
\hline
\end{tabular}

Table 9 Performance evaluation with $\mathrm{FH}$

\begin{tabular}{cc}
\hline Channel model & IEEE 802.15 .4 PDR/\% \\
\hline A & $95 \pm 0.78$ \\
B & $90 \pm 2$ \\
C & $85 \pm 2$ \\
D & $90 \pm 1$ \\
\hline
\end{tabular}

\subsubsection{Channel Model B}

The PDR of $77 \%$ in a single channel, as presented in Table 8, improves to $90 \%$ in $\mathrm{FH}$, as presented in Table 9. The increase in PDR with FH is $13 \%$. This channel model exhibits a strong fading in $2400-2430 \mathrm{MHz}$ frequency band of $30 \mathrm{MHz}$ bandwidth, as there are 10 small and large fades in the frequency response. There are two deep fades in the frequency response, which cause a huge reduction in PDR when signals are transmitted at these frequencies. The PDR with a single channel in this channel model is less than the PDR using a single channel in Model A, as frequency selective fading is present in almost whole of 2 400-2 $430 \mathrm{MHz}$ frequency band. The PDR with FH as transmission method is $90 \%$ which is considered very good for this channel model, also the increase in PDR with FH is significant. When signals are transmitted using 16 channels with $83.5 \mathrm{MHz}$ bandwidth, then in this frequency range many more deep fades are presented. Thus, the PDR would be near $50 \%$ with a single channel and 0 in many channels, and in $80 \%-90 \%$ range with $\mathrm{FH}$.

\subsubsection{Channel Model C}

The PDR of $70 \%$ in a single channel, as presented in Table 8 , improves to $85 \%$ in $\mathrm{FH}$, as presented in Table 9 .
The increase in PDR with FH is 15\%. This channel model shows a very strong frequency selective fading in $2400-$ $2430 \mathrm{MHz}$ frequency band of $30 \mathrm{MHz}$ bandwidth, as there are three very deep fades and many large fades in the frequency response. As there are multiple deep fades in the frequency response, they cause a huge reduction in PDR when signals are transmitted at these frequencies.

The PDR with a single channel in this channel model is less than the corresponding PDRs in all the other channel models, as very strong frequency selective fading is presented across all of 2 400-2 $430 \mathrm{MHz}$ frequency band. The PDR with FH as the transmission method is $85 \%$ which can be considered very good for this channel model, also the increase in PDR with FH is significant. When signals are transmitted using 16 channels with $83.5 \mathrm{MHz}$ bandwidth, then in this frequency range many more deep fades are presented. Thus, the PDR will be less than 50\% with a single channel and 0 in many channels, and in $75 \%-$ $85 \%$ range with $\mathrm{FH}$.

\subsubsection{Channel Model D}

The PDR of $80 \%$ in a single channel, as presented in Table 8, improves to $90 \%$ in $\mathrm{FH}$, as presented in Table 9. The increase in PDR with FH is $10 \%$. This channel model shows a flat fading in $2400-2430 \mathrm{MHz}$ frequency band of $30 \mathrm{MHz}$ bandwidth, as there are three noticeable fades in the frequency response, one fade is big while the other two are small. These three fades together are a major source of reduction in PDR when only a single channel is used. The signal fading in this channel model is more than the fading in Model A, but less than the fading in Model B and Model C. The PDR with FH as the transmission method is $90 \%$ which is considered very good for any channel model, also the increase in PDR with FH is significant. When signals are transmitted using 16 channels with $83.5 \mathrm{MHz}$ bandwidth, then in this frequency range eight more deep fades are presented. Thus, the PDR will be less than $50 \%$ with a single channel and 0 in many channels, and in $80 \%-90 \%$ range with $\mathrm{FH}$.

We have tested the performance of FH in many different wireless channel models, such as flat fading and frequencyselective fading channels. Our results and analysis show that $\mathrm{FH}$ achieves a PDR of $85 \%$ and more in all scenarios. The difference of PDR between FH and the single channel is very noticeable, as increase in PDR with FH is at least $10 \%$ and mostly much more than $10 \%$.

\section{Conclusions}

In this work we analyze the performance of FH in IEEE 802.15.4 based WSNs. We show that IEEE 802.11.b/g de- 
vices indeed cause a great interference to IEEE 802.15.4 devices. We evaluate the performance of IEEE 802.15.4 networks using a single channel and the FH method, when IEEE $802.11 . \mathrm{b} / \mathrm{g}$ devices are also transmitting on the same frequency in the same area. It is demonstrated that under the strong interference the PDR of IEEE 802.15.4 networks with the single channel is less than $50 \%$, while this PDR is greater than $90 \%$ with FH. If the distance between sensor nodes is increased, then the PDR goes down to $10 \%$ for the single channel and for FH it only decreases to $85 \%$. FH in IEEE 802.15.4 using free channels $(15,20,25,26)$ has been proposed and examined critically. In all cases, the PDR is in the range of $98 \%-99 \%$. This reduced channel set technique is most useful against the IEEE 802.11.b interferer. In the last part of this work, we evaluate the performance of IEEE 802.15.4 networks in fading channels for single frequency and FH. The performance improvement with FH over the single channel in fading channels is at least $10 \%$ and mostly much more than $10 \%$. Therefore, FH is a very useful data transmission method for IEEE 802.15.4 devices to overcome the interference and fading channels.

\section{References}

[1] IEEE Std. 802.15.4. Wireless medium access control (MAC) and physical layer (PHY) specifications for low-rate wireless personal area networks (WPANs). Piscataway, N.J.: IEEE, 2006.

[2] IEEE Std. 802.11. Wireless LAN medium access control (MAC) and physical layer (PHY) specifications. Piscataway, N.J.: IEEE, 1999.

[3] JANG S, PARK L, NA W, et al. Optimization of ISM band interference coordination between WLAN and IEEE 802.15.4 using NAV on PAN coordinator. Proc. of the 8th International Conference on Ubiquitous and Future Networks, 2016: 688 690.

[4] GUO W, HEALY W M, ZHOU M C. Impacts of 2.4-GHz ISM band interference on IEEE 802.15.4 wireless sensor network reliability in buildings. IEEE Trans. on Instrumentation \& Measurement, 2012, 61(9): 2533-2544.

[5] LAURIDSEN M, VEJLGAARD B, KOVACS I Z, et al. Interference measurements in the European $868 \mathrm{MHz}$ ISM band with focus on LoRa and SigFox. Proc. of the IEEE Wireless Communications and Networking Conference, 2017: 1-6.

[6] AZMI N, KAMARUDIN L M, MAHMUDDIN M, et al. Interference issues and mitigation method in WSN 2.4GHz ISM band: a survey. Proc. of the 2nd International Conference on Electronic Design, 2014: 403-408.

[7] MUCCHI L, CARPINI A. Aggregate interference in ISM band: WBANs need cognitivity? Proc. of the 9th International Conference on Cognitive Radio Oriented Wireless Networks and Communications, 2014: 247-253.

[8] MUCCHI L, CARPINI A. ISM band aggregate interference in BAN-working environments. Proc. of the 8th International Symposium on Medical Information and Communication Technology, 2014: 1-5.

[9] GIRI M K, TIWARI G. A channel utilization scheme for IEEE 802.15.4 working on ISM band under IEEE 802.11 interfer- ence. Proc. of the 3rd International Conference on Computing for Sustai, 2016: 806-810.

[10] TIWARI G, GIRI M K. A framework for throughput measurement of IEEE 802.15.4 under the IEEE 802.11 interference working on $2.4 \mathrm{GHz}$ ISM band. Proc. of the 3rd International Conference on Signal Processing and Integrated Networks, 2016: $348-352$.

[11] STANIEC K. IEEE 802.15.4 (ZigBee) immunity to in-band interference and multipath propagation. Proc. of the Radio and Antenna Days of the Indian Ocean, 2015: 1-2.

[12] ESEMANN T, HELLBRUECK H. In-band interference detection on reception for IEEE 802.15.4 transmissions. Proc. of the European Wireless, 2015: 1-6.

[13] ZACHARIAS S, NEWE T, O'KEEFFE S, et al. 2.4 GHz IEEE 802.15.4 channel interference classification algorithm running live on a sensor node. Sensors, 2012, 3(11): 1-4

[14] VELAGAPUdi P, ERAVATRI B C, MANTRI M B. Performance analysis of various IEEE 802.15.4 PHYs under Rayleigh fading channel. Proc. of the International Conference on Advanced Computing and Communication Systems, 2013: $1-5$.

[15] MARCO P D, FISCHIONE C, SANTUCCI F, et al. Modeling IEEE 802.15.4 networks over fading channels. IEEE Trans. on Wireless Communications, 2015, 13(10): 5366-5381.

[16] MANTRI M B, VELAGAPUDI P, ERAVATRI B C. Performance analysis of $2.4 \mathrm{GHz}$ IEEE $802.15 .4 \mathrm{PHY}$ under various fading channels. Proc. of the International Conference on Emerging Trends in Communication, Control, Signal Processing and Computing Applications, 2013: 1-4.

[17] MARCO P D, FISCHIONE C, SANTUCCI F, et al. Effects of Rayleigh-lognormal fading on IEEE 802.15.4 networks. Proc. of the IEEE International Conference on Communications, 2013: 1666 - 1671

[18] NITHYA V, RAMACHANDRAN B, BHASKAR V. Energy and error analysis of IEEE 802.15.4 Zigbee RF transceiver under various fading channels in wireless sensor network. Proc. of the 4th International Conference on Advanced Computing, 2012: $1-5$.

[19] NITHYA V, RAMACHANDRAN B, BHASKAR V. BER evaluation of IEEE 802.15.4 compliant wireless sensor networks under various fading channels. Wireless Personal Communications, 2014, 77(4): 3105-3124.

[20] NITHYA V, RAMACHANDRAN B, BHASKAR V. Energy efficient coded communication for IEEE 802.15.4 compliant wireless sensor networks. Wireless Personal Communications, 2014, 77(1): $675-690$.

[21] FANG S, BERBER S M, SWAIN A K. Energy consumption evaluations of cluster-based sensor nodes with IEEE 802.15.4 transceiver in flat Rayleigh fading channel. Proc. of the International Conference on Wireless Communications \& Signal Processing, 2009: 1-5.

[22] HAN S, LEE S, LEE S, et al. Coexistence performance evaluation of IEEE 802.15.4 under IEEE 802.11B interference in fading channels. Proc. of the 18th IEEE International Symposium on Personal, Indoor and Mobile Radio Communications, 2007: $1-5$.

[23] MANKAR D, CHAUDHARI B S. Dynamic performance analysis of IEEE 802.15.4 devices under various RF interferences. Proc. of the International Conference on Inventive Computation Technologies, 2016: 1-4.

[24] NOREEN U, BOUNCEUR A, CLAVIER L, et al. Performance evaluation of IEEE 802.15.4 PHY with impulsive network interference in cupcarbon simulator. Proc. of the International Symposium on Networks, Computers and Communica- 
tions, 2016: $1-6$.

[25] KHAN S, T KRISHNAN S S, KOTHARI S, et al. Interference study in adjacent and alternate channels of IEEE 802.15.4 spectrum. Proc. of the International Conference on Wireless Communications, Signal Processing and Networking, 2016: $391-395$.

[26] YAALA S B, THÉOLEYRE F, BOUALLEGUE R. Performance study of co-located IEEE 802.15.4-TSCH networks: interference and coexistence. Proc. of the IEEE Symposium on Computers and Communication, 2016: 513-518.

[27] JI G, WANG Z, ZHU D, et al. Performance evaluation and improvement of TCP/IPv6 over IEEE 802.15.4 under Wi-Fi interference. Proc. of the 28th IEEE Canadian Conference on Electrical and Computer Engineering, 2015: 1095-1100.

[28] TYTGAT L, YARON O, POLLIN S, et al. Analysis and experimental verification of frequency-based interference avoidance mechanisms in IEEE 802.15.4. IEEE/ACM Trans. on Networking, 2015, 23(2): 369-382.

[29] NAGAI Y, HORI T, YOKOYAMA Y, et al. Advanced wireless cooperation mechanisms for interference mitigation in the $2.4 \mathrm{GHz}$ ISM band. Proc. of the IEEE Consumer Communications and Networking Conference, 2012: 364-365.

[30] CUZANAUSKAS T, MEDEISIS A, HADDAD Y, et al. Interference-aware power coordination game for ISM bands. Proc. of the 9th International Conference on Cognitive Radio Oriented Wireless Networks and Communications, 2014: $389-394$.

[31] HITHNAWI A, LI S, SHAFAGH H, et al. CrossZig: combating cross-technology interference in low-power wireless networks. Proc. of the 15th ACM/IEEE International Conference on Information Processing in Sensor Networks, 2016: 1-12.

[32] PATEL N R, KUMAR S. Enhanced clear channel assessment for slotted CSMA/CA in IEEE 802.15.4. Wireless Personal Communications, 2017, 95(4): $4063-4081$.

[33] CHEN S, SUN T, YUAN J, et al. Performance analysis of IEEE 802.15.4e time slotted channel hopping for low-rate wireless networks. KSII Transactions on Internet \& Information Systems, 2013, 7(1): 1-21.

[34] CHOI K, CHUNG S H. Enhanced time-slotted channel hopping scheduling with quick setup time for industrial Internet of Things networks. International Journal of Distributed Sensor Networks, 2017, 13(6): 155014771771362.

[35] GANERIWAL S, KUMAR R, SRIVASTAVA M B. Timingsync protocol for sensor networks. Proc. of the International Conference on Embedded Networked Sensor Systems, 2003: $138-149$.

[36] CARSTEN B, ANGELO P C, ZACH S. CoAP: an application protocol for billions of tiny internet nodes. IEEE Internet Computing, 2012, 16(2): 62-67.

[37] TAO Y, SERHIY B, JEAN-MARK L P, et al. Dynamic phasor modeling of multi-generator variable frequency electrical power systems. IEEE Trans. on Power Systems, 2016, 31(1): $563-571$.

[38] ZHANG J J, HUANG Y M, SHI Q J, et al. Codebook design for beam alignment in millimeter wave communication systems. IEEE Trans. on Communications, 2017, 65(11): 4980 4995.

[39] VANGELIS A, STEFANOS P, VASILIOS A S, et al. Adjacent channel interference in 802.11a is harmful: testbed validation of a simple quantification model. IEEE Communications Magazine, 2011, 49(3): 160-166.

[40] DON T. Principles of spread-spectrum communication systems. Springer US, 2005, 3(5): 45-45.

[41] KHUN-JUSH J, SCHRAMM P, WACHSMANN U, et al. Structure and performance of the HIPERLAN/2 physical layer. Proc. of the Vehicular Technology Conference, 1999: 1-5.

\section{Biographies}

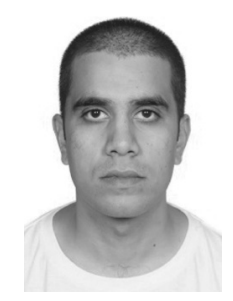

JAVED Umer received his B.S. degree in electrical engineering from University of Engineering \& Technology Taxila, Taxila, Pakistan, in 2006, and M.S. degree in communications engineering from Helsinki University of Technology, Espoo, Finland, in 2009. He is currently studying for his Ph.D. degree at Shanghai Jiao Tong University, Shanghai, China.

E-mail: umerjaved@sjtu.edu.cn

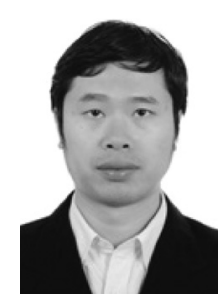

HE Di received his Ph.D. degree from Shanghai Jiao Tong University in 2002. From 2002 to 2004, he was a postdoctoral fellow in the Department of Electrical and Computer Engineering, University of Calgary, Canada. From 2004 to present, he has been an associate professor at Shanghai Jiao Tong University. His research interests include wireless positioning, wireless communications and wireless signal processing.

E-mail: dihe@sjtu.edu.cn

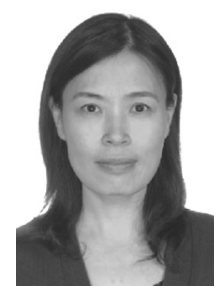

LIU Peilin received her Ph.D. degree majoring in electronic engineering from the University of Tokyo, Tokyo, Japan, in 1998. From 1999 to 2003, she was a senior researcher at the Central Research Institute of Fujitsu, Tokyo. She is currently a professor in the Department of Electronic Engineering, Shanghai Jiao Tong University, an executive director in Shanghai Key Laboratory of Navigation and Location-based Service, and is responsible for a series of important projects, such as Beidou system, system-on-chip platform development, low-power and high-performance communication digital signal processing. Her research interests include signal processing, low-power computing architecture, and application-oriented SoC design and verification. E-mail: liupeilin@sjtu.edu.cn

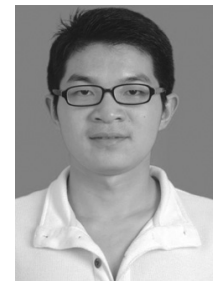

YANG Yueming received his M.E. degree from Fudan University in 2012. From 2013 to present, he has been a chip design manager at Shanghai Panchip Microelectronics Co., Ltd. His research interests include wireless communication, high performance and low power SoC design and implementation. E-mail: yangyueming@panchip.com 